\title{
Variability of adjacency effects in sky reflectance measurements
}

\author{
Philipp M. M. Groetsch ${ }^{1,2, *}$, Peter Gege ${ }^{3}$, Stefan G. H. Simis ${ }^{4}$, Marieke A. Eleveld ${ }^{5,2}$, And \\ Steef W. M. Peters ${ }^{1}$ \\ ${ }^{1}$ Water Insight, Marijkeweg 22, 6709 PG Wageningen, The Netherlands \\ ${ }^{2}$ Vrije Universiteit Amsterdam, De Boelelaan 1087 - 1081 HV Amsterdam, The Netherlands \\ ${ }^{3}$ DLR German Aerospace Center, Münchener Straße 20, 82234 Weßling, Germany \\ ${ }^{4}$ Plymouth Marine Laboratory, Prospect Place, The Hoe, PL1 3DH Plymouth, United Kingdom \\ ${ }^{5}$ Deltares, P.O. Box 177, $2600 \mathrm{MH}$ Delft, The Netherlands \\ *Corresponding author: groetsch@waterinsight.nl
}

Compiled July 31, 2017

Sky reflectance $R_{s k y}(\lambda)$ is used to correct in situ reflectance measurements in the remote detection of water colour. We analysed the directional and spectral variability in $R_{s k y}(\lambda)$ due to adjacency effects against an atmospheric radiance model. The analysis is based on one year of semi-continuous $R_{s k y}(\lambda)$ observations that were recorded in two azimuth directions. Adjacency effects contributed to $R_{s k y}(\lambda)$ dependent on season and viewing angle, and predominantly in the near-infrared (NIR). For our test area, adjacency effects spectrally resembled a generic vegetation spectrum. The adjacency effect was weakly dependent on the magnitude of Rayleigh- and aerosol-scattered radiance. Reflectance differed between viewing directions $5.4 \pm 6.3 \%$ for adjacency effects and $21.0 \pm 19.8 \%$ for Rayleigh- and aerosol-scattered $R_{s k y}(\lambda)$, in the NIR. It is discussed under which conditions in situ water reflectance observations require dedicated correction for adjacency effects. We provide an open source implementation of our method to aid identification of such conditions. (c) 2017 Optical Society of America

OCIS codes: (010.0010) Atmospheric and oceanic optics; (280.0280) Remote sensing and sensors; (010.1285) Atmospheric correction; (010.5620) Radiative transfer; (010.7340) Water;

\section{http://dx.doi.org/10.1364/ao.XX.XXXXXX}

Coastal and inland water bodies are challenging targets for satellite remote sensing compared to the open ocean, due to higher optical complexity of water and atmosphere, small spatial footprints, and adjacency effects [1]. Adjacent land can increase the remotely sensed water leaving radiance signal when contrasts are present in ground albedo $r_{g}(\lambda)$, i.e. the fraction of global incident solar irradiance that is reflected back into space [2]. Adjacency effects are negligible for most open-ocean environments due to low $r_{g}(\lambda)$ contrasts at sea [3]. Land cover (e.g. vegetation, ice/snow, roads) reflectance typically exceeds that of water by an order of magnitude and may thus be expected to contribute to downwelling irradiance and observed waterleaving radiance as the result of (multiple-) scattering processes in the atmosphere [4].

Various adjacency correction approaches exist for satellite or airborne remote sensing observations for water close to land [e.g. 5-7]. Calibration and validation of the adjacency correction rely heavily on in situ reference measurements, such as remote sensing reflectance $R_{r s}(\lambda) . R_{r s}(\lambda)$ is defined as the ratio of water leaving radiance $L_{w}(\lambda)$ to downwelling irradiance $E_{d}(\lambda)$. For isotropic (Lambertian) reflection, normalization with $E_{d}(\lambda)$ at water surface level should account for any adjacency components in the light field [8]. However, $L_{w}(\lambda)$ cannot be observed directly from above the water due to reflections at the surface [9]. A common approach to derive $L_{w}(\lambda)$ from upwelling radiance $L_{u}(\lambda)$ is to subtract a fraction $\rho$ of sky radiance $L_{s k y}(\lambda)$ in specular direction of the sea-viewing sensor [10]:

$$
R_{r s}(\lambda)=\frac{L_{u}(\lambda)}{E_{d}(\lambda)}-\rho \frac{L_{s k y}(\lambda)}{E_{d}(\lambda)} .
$$

Radiance sensor azimuth angles are assumed equal, with zenith angles mirrored in the horizontal plane. In the following we focus on the correction term, $L_{s k y}(\lambda) / E_{d}(\lambda)$, which is referred to as sky reflectance $R_{\text {sky }}(\lambda)$. Natural water surfaces are windroughened and therefore reflect radiance also from outside the $L_{\text {sky }}(\lambda)$ sensor field-of-view [e.g. 10, Fig. 2]. A measurement of $L_{s k y}(\lambda)$ is thus geometrically not representative of the radiance reflected at a wind-roughened water surface. As long as reflected radiance is spectrally equivalent to measured $L_{s k y}(\lambda)$, $R_{r s}(\lambda)$ can still be accurately derived with eq. (1) and a suitable choice for $\rho$ [e.g. 11, 12]. If this is not the case, spectrally variable contributions to $L_{w}(\lambda)$ by sun and sky glint (Rayleigh- or aerosol-scattered) may be accounted for with bio-optical modelling [e.g. 13]. The adjacency component of the downwelling light field depends on $r_{g}(\lambda)$, atmospheric composition, illumination conditions, and viewing geometry $[14,15]$ and is expected to be variable in intensity and spectral shape, for different viewing angles. To date, no methodology is available to identify in situ 
reflectance measurements that are contaminated by adjacency effects.

In this letter, we assess directional and spectral variability in $R_{s k y}(\lambda)$ due to the adjacency effect against an atmospheric model. The model is used to partition $R_{s k y}(\lambda)$ into two components: sky reflectance that has not interacted with ground cover, and sky reflectance due to adjacency effects. The procedure was applied to one year of semi-continuous $R_{s k y}(\lambda)$ observations recorded in two azimuth directions, to asses the spatio-temporal variability of each component. Subsequently, $r_{g}(\lambda)$ of the sensor environment was derived to assess spectral variability.

Semi-continuous measurements were recorded with a fixedposition reflectance instrument in the eutrophic Lake Paterswolde (Paterswoldsemeer, the Netherlands, $53.1631^{\circ} \mathrm{N}$, $\left.6.5773^{\circ} \mathrm{E}\right)$. Trees and fields dominated the surroundings of the southern part of the lake where the instrument was located, with the city of Groningen to the North. The nearest vegetation was located approximately $50 \mathrm{~m}$ away from the sensors. The instrument carried two sets of inter-calibrated $L_{u}\left(\lambda, \theta_{\text {view }}, \phi\right), L_{s k y}\left(\lambda, \theta_{\text {view }}^{\prime}, \phi\right)$, and $E_{d}(\lambda)$ channels $(400-880$ $\mathrm{nm})$, with azimuth angles $\phi$ pointing north-northeast (NNE) and north-northwest (NNW), respectively. Viewing zenith angles $\theta_{\text {view }}^{\prime}, \theta_{\text {view }}$ of sky and upwelling radiance sensors were fixed at $40^{\circ}$ from zenith and nadir, respectively. The instrument was deployed from 5 September 2015 until 3 October 2016, excluding November to January. During the deployment, clear sky conditions between 9 and $15 \mathrm{~h}$ local time occurred for at least one measurement on 229 days and 4542 observations in total. The sky was considered clear if $L_{s k y} / E_{d}(750 \mathrm{~nm})<0.05$ [11] in both azimuthal viewing directions. We did not consider variability in $L_{u}(\lambda)$ in this analysis.

We utilize an analytical parametrisation of downwelling radiation by [3] that was spectrally expanded by [16], further referred to as GC90. The model describes $E_{d}(\lambda)$ as the sum of direct $E_{d d}(\lambda)$ and diffuse $E_{d s}(\lambda)$ irradiance, with the latter composed of Rayleigh- and aerosol-scattered irradiances $\left(E_{d s r}(\lambda)\right.$ and $E_{d s a}(\lambda)$, respectively). The influence of clouds on $E_{d}(\lambda)$ is not included. GC90 is based on earlier work by [17], modified to yield irradiances typically observed under maritime atmospheres, where adjacency effects may safely be ignored. This assumption may not be valid for measurements in lakes or coastal areas. Hence we adapted adjacency irradiance $E_{d s g}(\lambda)$, as formulated by [17], for use with GC90. It is assumed that a fraction $r_{g}(\lambda)$ of $E_{d}(\lambda)$ is reflected at the ground, and subjected to Rayleigh- and aerosol scattering that return a fraction $r_{s}(\lambda)$ (sky reflectivity) towards a sky-viewing observer [17]:

$$
E_{d s g}(\lambda)=E_{d}(\lambda) r_{g}(\lambda) r_{s}(\lambda) \times\left[1-r_{s}(\lambda) r_{g}(\lambda)\right]^{-1} .
$$

Sky reflectivity is calculated based on atmospheric transmission coefficients and aerosol forward scattering probability $F_{a}$ :

$$
\begin{aligned}
r_{s}(\lambda) & =T_{o}(\lambda) T_{w v}(\lambda) T_{a a}(\lambda) \\
& \times\left[0.5\left(1-T_{r}(\lambda)\right)+\left(1-F_{a}\right) T_{r}(\lambda)\left(1-T_{a s}(\lambda)\right)\right],
\end{aligned}
$$

with reduced transmission due to oxygen absorption $T_{0}(\lambda)$, water vapour absorption $T_{w v}(\lambda)$, aerosol absorption $T_{a a}(\lambda)$, Rayleigh-scattering $T_{r}(\lambda)$, and aerosol scattering $T_{a s}(\lambda)$. A factor $\pi^{-1}$ is assumed to express modelled irradiances in units of radiance. Modelled sky reflectance $R_{s k y}^{m}(\lambda)$ is thus calculated as:

$$
R_{s k y}^{m}(\lambda)=\frac{f_{d s r} L_{d s r}(\lambda)+f_{d s a} L_{d s a}(\lambda)+f_{d s g} L_{d s g}(\lambda)}{E_{d d}(\lambda)+E_{d s r}(\lambda)+E_{d s a}(\lambda)+E_{d s g}(\lambda)}
$$

with scaling factors $f$ and radiances $L$ for Rayleigh- $(d s r)$, aerosol$(d s a)$, and adjacency-scattering $(d s g)$, respectively. Further detail on the calculation of these parameters is given in [3], [17], and [16]. Least-squares minimization (bounded Nelder-Mead) of the residual-sum-of-squares (RSS) between observed $R_{\text {sky }}(\lambda)$ and modelled $R_{s k y}^{m}(\lambda)$,

$$
R S S=\sum^{i}\left(R_{s k y}\left(\lambda_{i}\right)-R_{s k y}^{m}\left(\lambda_{i}\right)\right)^{2} \times W\left(\lambda_{i}\right),
$$

was carried out with bounded parameters as listed in Table 1 , air mass type fixed to 1 , standard atmospheric pressure of 1013.25 mbar, relative humidity of $60 \%$, ozone scale height of $0.3 \mathrm{~cm}$, water vapour scale height of $2.5 \mathrm{~cm}$, and weighting vector $W(\lambda)=1$. A fit was regarded successful if RSS $<0.01$ $s r^{-1}$, which was the case for 4404 of 4542 observations. The 'lawn/grass' spectrum from the SMARTS2 model [18] gave the lowest average RSS and was used as reference $r_{g}(\lambda)$ in further calculations (see Fig. 3, $r_{g}^{\text {Grass }}$ ).

Table 1. Average (mean) fit results and standard deviations (4404 fits) for bounded model parameters: Rayleigh-scattering irradiance factor $f_{d s r}$, aerosol-scattering irradiance factor $f_{d s a}$, adjacency irradiance factor $f_{d s g}$, Ångström exponent $\alpha$, turbidity coefficient $\beta$. Optimization was carried out with and without taking adjacency effects into account, and resulted in normalized root-mean-square deviations (NRMSD).

Param. Start (bounds) No adjacency With adjacency

\begin{tabular}{lrrr}
\hline \hline$f_{d s r},[-]$ & $1(0-10)$ & $0.80 \pm 0.12$ & $0.80 \pm 0.13$ \\
$f_{d s a},[-]$ & $1(0-10)$ & $1.80 \pm 2.33$ & $0.98 \pm 1.77$ \\
$f_{d s g,},[-]$ & $1(0-10)$ & - & $0.62 \pm 0.28$ \\
$\alpha,[-]$ & $1(0-3)$ & $1.64 \pm 0.65$ & $1.30 \pm 0.65$ \\
$\beta,[-]$ & $0.05(0-1)$ & $0.08 \pm 0.13$ & $0.17 \pm 0.22$ \\
\hline NRMSD, [\%] & & $7.13 \pm 2.87$ & $1.29 \pm 0.29$ \\
\hline
\end{tabular}

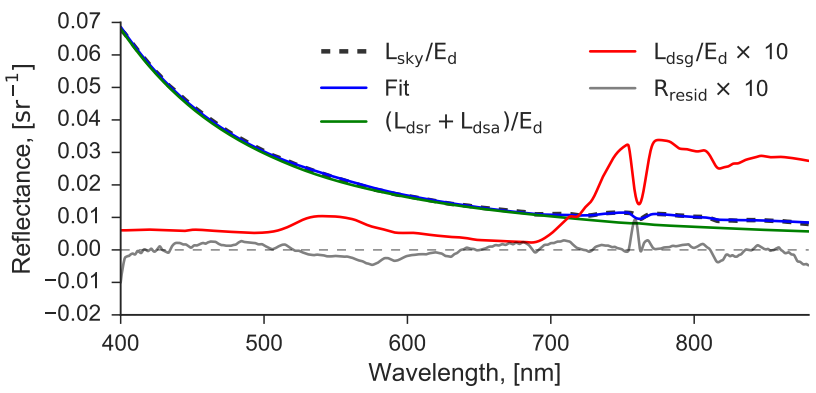

Fig. 1. Fit curve, diffuse $\left(L_{d s r}(\lambda)+L_{d s a}(\lambda)\right) / E_{d}(\lambda)$ and adjacency $\left.L_{d s g}(\lambda)\right) / E_{d}(\lambda)$ sky reflectance components, and residual spectrum $R_{\text {resid }}(\lambda)$, derived from model optimization to a $L_{s k y}(\lambda) / E_{d}(\lambda)$ observation.

$R_{\text {sky }}(\lambda)$ is representative of molecular Rayleigh- and aerosolscattered light and contains no atmospheric absorption features [19], if recorded under clear sky conditions and in absence of adjacency effects. Reflectance of vegetation is typically low for 


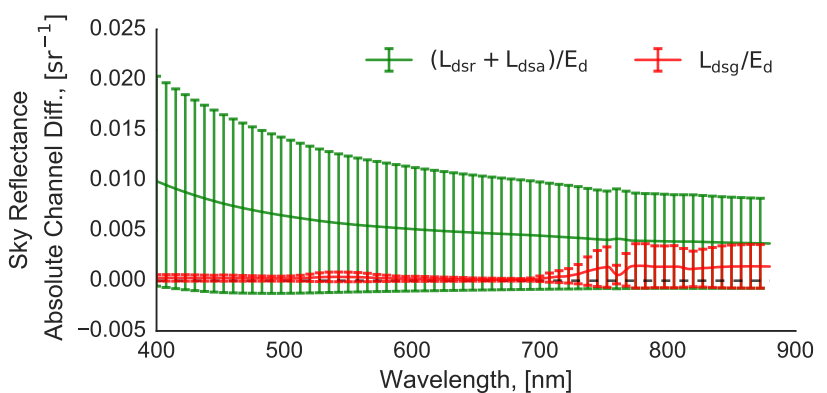

Fig. 2. Mean absolute differences (standard deviation depicted as error bars) between modelled sky reflectance components in direction NNW and NNE

wavelengths below approximately $680 \mathrm{~nm}$ from where it rises steeply - the so-called red edge of vegetation [20]. This was exploited to compare model performance with, and without taking adjacency effects into account. For the latter, RSS was minimized in the spectral region 400 to $680 \mathrm{~nm}$ (weighting vector $W(\lambda>680 \mathrm{~nm})=0,1$ elsewhere) and $f_{d s g}=0$ (for all bounded parameters, see Table 1$)$.

Figure 1 shows model fit results for a typical summer observation of $R_{s k y}(\lambda)$ above Paterswoldsemeer. The fit curve is almost indistinguishable from the $L_{s k y} / E_{d}$ observation (normalized root-mean-square deviation NRMSD of $0.97 \%$ ), also in the near-infrared (NIR) spectral bands above $680 \mathrm{~nm}$ where adjacency effects due to vegetation are expected, and around the oxygen A-band rotational absorption lines near $762 \mathrm{~nm}$. Model optimization in the 400-680 $\mathrm{nm}$ spectral region and without correcting for adjacency effects resulted in a NRMSD of 6.8 $\%$ (400-880 nm). However, Rayleigh- and aerosol-scattered reflectance matches the $L_{s k y} / E_{d}$ observation well up to approximately $680 \mathrm{~nm}$, supporting that adjacency effects predominantly affected the NIR bands for our test data. Model fits (with and without accounting for adjacency effects) were repeated for all sky reflectances in the data set, and parameter results are summarized in Table 1 . Accounting for adjacency effects reduced average NRMSD more than five-fold. Variability of $f_{d s g}$ suggests strong seasonality: in February, $f_{d s g}$ averaged lowest to $0.35 \pm 0.16$ (NNE) and $0.34 \pm 0.15$ (NNW), compared to maximum values in June of $0.91 \pm 0.30$ (NNE) and $0.87 \pm 0.26(\mathrm{NNW})$. Figure 2 shows that NNE- and NNWpointing channels differed more strongly in terms of Rayleighand aerosol contributions than adjacency effects. The ratio $L_{d s g}(\lambda) /\left[L_{d s r}(\lambda)+L_{d s a}(\lambda)\right]$ averaged $0.13 \pm 0.14$ and was less pronounced in the visible spectral range $(0.04 \pm 0.01,400-680$ $\mathrm{nm})$ compared to the NIR $(0.26 \pm 0.13,680-880 \mathrm{~nm})$. When compared to observed $R_{s k y}(\lambda)$, directional reflectance differences in the NIR averaged to $5.4 \pm 6.3 \%$ (adjacency) and $21.0 \pm 19.8 \%$ (Rayleigh- and aerosol-scattered). Weak correlations were found between $f_{d s r}$ and $f_{d s g}\left(R^{2}<0.20, p \ll 0.05\right.$, both directions), $f_{d s a}$ and $f_{d s g}\left(R^{2}<0.07, p \ll 0.05\right.$, both directions), and between viewing directions for $f_{d s g}\left(R^{2}=0.42, p \ll 0.05\right)$.

It is concluded that adjacency effects in this study contributed to observed $R_{\text {sky }}(\lambda)$ a) dependent on season and viewing angle, b) predominantly in the NIR, c) and weakly dependent on Rayleigh- and aerosol-scattered reflectance.

Thus far, $r_{g}(\lambda)$ was approximated by scaling a constant vegetation reference albedo from literature. From above, it is obvious

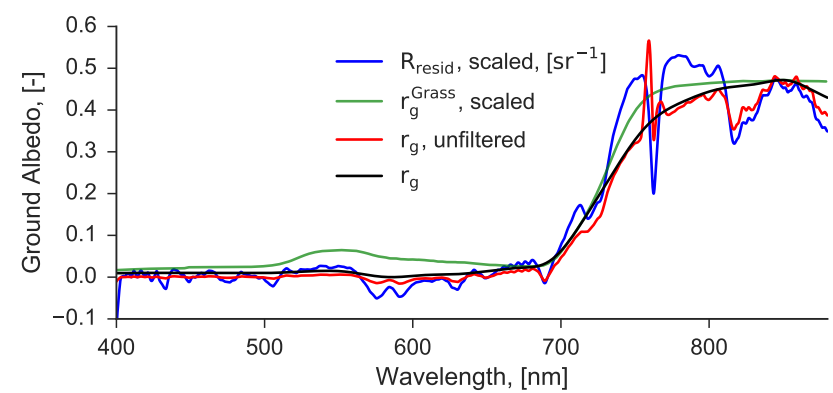

Fig. 3. Ground albeo $r_{g}(\lambda)$, derived from optimization residuals $R_{\text {resid }}(\lambda)$. Reference ground albedo $r_{g}^{G r a s s}(\lambda)$ is plotted for visual comparison (scaled to match $r_{g}(\lambda)$ peak height).

that this scaling will be spatiotemporally variable. We subsequently investigated whether $r_{g}(\lambda)$ was variable not only in magnitude but also in spectral shape. Based on b), adjacency effects were ignored in a second round of model fits that excluded the NIR spectral range. Corresponding residual spectra $R_{\text {resid }}(\lambda)$,

$$
R_{\text {resid }}(\lambda)=\frac{L_{s k y}(\lambda)}{E_{d}(\lambda)}-\frac{L_{s}^{m}(\lambda)}{E_{d}^{m}(\lambda)},
$$

over the complete spectral range $(400-880 \mathrm{~nm})$ were thus assumed to be exclusively caused by adjacency effects. This assumption allowed to solve eq. (4) for spectral ground albedo $r_{g}(\lambda)$

$$
r_{g}(\lambda) \approx \frac{\pi}{f_{d s g}} \times \frac{R_{r e s i d}(\lambda)}{r_{s}(\lambda)} .
$$

Equation 7 was applied to all $R_{\text {resid }}(\lambda)$ to calculate $r_{g}(\lambda)$ for the whole data set. Residual absorption features in derived $r_{g}(\lambda)$ due to modelling imperfections of water vapour absorption (710 - $735 \mathrm{~nm}$ and 805 - $840 \mathrm{~nm}$ ) and oxygen absorption (755 - 770 $\mathrm{nm}$ ) were removed and linearly interpolated before smoothing with a Hamming window of $50 \mathrm{~nm}$ width, while residuals due to comparatively weak and broad ozone absorption were not corrected for. Derived $r_{g}(\lambda)$ also accounted for scaling effects, thus $f_{d s g}$ was fixed to 1 . $R_{\text {resid }}(\lambda)$ may be negative for some bands, which would lead to negative values for $r_{g}(\lambda)$. Since no information about absolute reflectance contrasts was available, $r_{g}(\lambda)$ spectra were shifted such that they became non-negative throughout the covered spectral range. An example of resulting $r_{g}(\lambda)$ is depicted in Fig. 3 and monthly averages are shown in Fig. 4. Ground albedo magnitude followed the seasonal vegetation cycle, with lower values in late autumn and early spring, and highest values in summer. This resembles variability in $f_{d s g}$ that was derived from model optimization with a fixed reference ground albedo. Mean $r_{g}(N I R)$ varied by $66.9 \%$ (NNE) and 64.2 $\%$ (NNW) (relative standard deviation). After removing scaling variability (division by mean), spectral variability in $r_{g}(N I R)$ of $13.4 \pm 7.8 \%$ (NNE) and $14.6 \pm 8.5 \%$ (NNW) (mean relative standard deviation) remained. Mean $r_{g}(N I R)$ differed (absolute differences) between NNE and NNW on average by $0.17 \pm 0.21$ for individual observations, $0.12 \pm 0.15$ for daily averages, and $0.02 \pm 0.01$ for monthly averages. Spectral shapes of derived ground albedo generally resembled that of $r_{g}^{\text {Grass }}(\lambda)$, though with albedo peak wavelength migrating from approximately 820 $\mathrm{nm}$ in spring and autumn to approximately $870 \mathrm{~nm}$ in summer.

In summary, short-term and seasonal variability in $R_{s k y}(\lambda)$ 

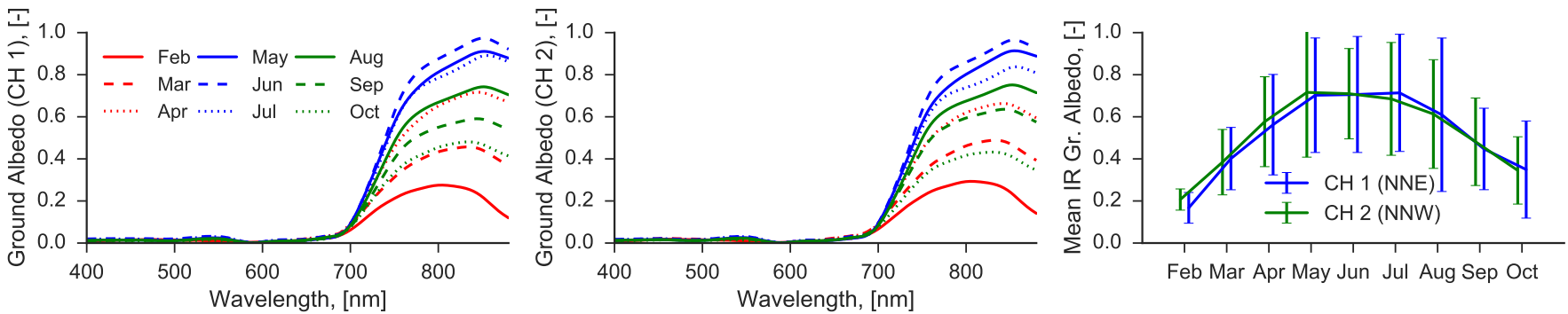

Fig. 4. Monthly averaged spectral ground albedos for azimuth directions NNE (left panel) and NNW (middle panel), and their average values in the 680-880 $\mathrm{nm}$ spectral range (right panel, error bars depict standard deviations).

due to adjacency effects were dominated by amplitude rather than spectral variation. Adjacency effect directionality is apparent from systematic amplitude differences in adjacency radiance towards NNE and NNW, and is further indicated by weak correlations between $f_{d s g}$ for these directions. Adjacency effects contributed primarily to the $R_{s k y}(\lambda)$ variability in the NIR, where accurate reflectance references are crucial for atmospheric correction and quality control of water-leaving radiance [11]. These results support that illumination conditions have a noticeable effect on apparent spectral ground albedo, e.g. due to inhomogeneous bidirectional reflectance distribution functions (BRDFs) of surrounding vegetation [15]. Models for these complex interactions [e.g. 15] could augment approximated $r_{g}(\lambda)$ spectra, e.g. when adjacency effects are not confined to the NIR or by providing realistic reflectance offsets. A spectrally constant reference albedo (here: $r_{g}^{G r a s s}(\lambda)$ ) is considered sufficient to model adjacency effects in the context of correction for water surface reflections.

Combined directional variability of Rayleigh- and aerosolscattered sky light and adjacency effects averaged more than $26 \%$ of observed $R_{s k y}(\lambda)$ for the analysed data set in the NIR. Wind-roughened water surfaces regularly reflect light also from outside the $45^{\circ}$ azimuth difference range considered here [e.g 10, Fig. 2]. Because $R_{s k y}(\lambda)$ components have distinct spectral characteristics and vary independently throughout the sky, a correction of $R_{r s}(\lambda)$ with specular sky radiance (eq. 1) can introduce spectral biases [21]. Bio-optical modelling approaches already account for scalar biases [21] or spectral offsets due to sun glint, and directional differences in Rayleigh-, and aerosolscattered components [13]. Adjacency effects may be resolved by adding $L_{d s g}(\lambda) / E_{d}(\lambda)$ to these models, given a realistic spectral approximation for adjacent ground albedo. Such a correction may also be relevant for observations on open water where e.g. cyanobacterial surface scum, floating vegetation, or fronts, could introduce adjacency effects similar to land vegetation. Clouds reflect more efficiently than air molecules and aerosols, and are thus likely to enhance adjacency effects. Cloud cover is not treated by the GC90 model and thus results presented here are limited to clear sky observations. Further research is encouraged to remove this limitation. The presented approach allows automated probing of $R_{r s}(\lambda)$ data sets (that include sky radiance) for directional differences in adjacency effects, but also Rayleigh- and aerosol-scattered sky light. For this purpose, we recommend to routinely measure $R_{s k y}(\lambda)$ for a range of viewing directions. If $R_{\text {sky }}(\lambda)$ is only available in the specular direction to the sea-viewing sensor, fit results for $f_{d s g}$ indicate the intensity of adjacency effects. The minimisation procedure and all required modelling components were implemented in Python and are publicly available under open license LGPL [22]. Affected observations, e.g. $f_{d s g}>10 \%$ of $f_{d s r}$, should be flagged suspect until a suitable $R_{r s}(\lambda)$ correction approach becomes available, especially in the context of atmospheric correction and vicarious calibration/validation of satellite observations.

\section{FUNDING AND ACKNOWLEDGEMENTS}

European Commission H2020 TAPAS (678396). We thank the two anonymous reviewers for their constructive criticism.

\section{REFERENCES}

1. S. C. J. Palmer, T. Kutser, and P. D. Hunter, Remote Sensing of Environment 157, 1 (2015).

2. B. A. Wielicki, Science 308, 825 (2005).

3. W. Gregg and K. L. Carder, Limonology And Oceanography 35, 1657 (1990).

4. R. Santer and C. Schmechtig, Applied optics 39, 361 (2000).

5. S. Sterckx, E. Knaeps, and K. Ruddick, International Journal of Remote Sensing 32, 6479 (2011).

6. R. Santer, "The correction of the adjacency effects," Tech. rep. (2013).

7. V. Kiselev, B. Bulgarelli, and T. Heege, Remote Sensing of Environment 157, 85 (2015).

8. R. Richter, M. Bachmann, W. Dorigo, and A. Müller, IEEE Geoscience and Remote Sensing Letters 3, 565 (2006).

9. C. D. Mobley, Light and water: Radiative transfer in natural waters (Academic Press, 1994).

10. C. D. Mobley, Applied optics 38, 7442 (1999).

11. K. G. Ruddick, V. De Cauwer, Y.-J. Park, and G. Moore, Limnology and Oceanography 51, 1167 (2006).

12. S. G. H. Simis and J. Olsson, Remote Sensing of Environment 135, 202 (2013).

13. P. M. M. Groetsch, P. Gege, S. G. H. Simis, M. A. Eleveld, and S. W. M. Peters, Optics Express 25, 1 (2017).

14. M. O. Román, C. B. Schaaf, P. Lewis, F. Gao, G. P. Anderson, J. L. Privette, A. H. Strahler, C. E. Woodcock, and M. Barnsley, Remote Sensing of Environment 114, 738 (2010).

15. S. Cui, X. Zhen, Z. Wang, S. Yang, W. Zhu, X. Li, H. Huang, and H. Wei, Optics Letters 40, 3842 (2015).

16. P. Gege, Applied optics 51, 1407 (2012).

17. R. E. Bird and C. Riordan, Journal of climate and applied meteorology 25, 87 (1986).

18. C. A. Gueymard, Solar Energy 71, 325 (2001).

19. P. Gege and P. Groetsch, "A spectral model for correcting sun glint and sky glint," in "Proceedings of Ocean Optics XXIII," (2016).

20. D. N. H. Horler, M. Dockray, and J. Barber, International Journal of Remote Sensing 4, 273 (1983).

21. Z. Lee, Y.-h. Ahn, C. Mobley, and R. Arnone, Optics Express 18, 171 (2010).

22. P. M. M. Groetsch, "Sky Reflectance Adjacency Model," http://doi.org/10.5281/zenodo.836756 (2017). 


\section{FULL REFERENCES}

1. S. C. J. Palmer, T. Kutser, and P. D. Hunter, "Remote sensing of inland waters: Challenges, progress and future directions," Remote Sensing of Environment 157, 1-8 (2015).

2. B. A. Wielicki, "Changes in Earth's Albedo Measured by Satellite," Science 308, 825-825 (2005).

3. W. Gregg and K. L. Carder, "A simple spectral solar irradiance model for cloudless maritime atmospheres," Limonology And Oceanography 35, 1657-1675 (1990).

4. R. Santer and C. Schmechtig, "Adjacency effects on water surfaces: primary scattering approximation and sensitivity study." Applied optics 39, 361-375 (2000).

5. S. Sterckx, E. Knaeps, and K. Ruddick, "Detection and correction of adjacency effects in hyperspectral airborne data of coastal and inland waters: the use of the near infrared similarity spectrum," International Journal of Remote Sensing 32, 6479-6505 (2011).

6. R. Santer, "The correction of the adjacency effects," Tech. rep. (2013).

7. V. Kiselev, B. Bulgarelli, and T. Heege, "Sensor independent adjacency correction algorithm for coastal and inland water systems," Remote Sensing of Environment 157, 85-95 (2015).

8. R. Richter, M. Bachmann, W. Dorigo, and A. Müller, "Influence of the adjacency effect on ground reflectance measurements," IEEE Geoscience and Remote Sensing Letters 3, 565-569 (2006).

9. C. D. Mobley, Light and water: Radiative transfer in natural waters (Academic Press, 1994).

10. C. D. Mobley, "Estimation of the remote-sensing reflectance from abovesurface measurements." Applied optics 38, 7442-55 (1999).

11. K. G. Ruddick, V. De Cauwer, Y.-J. Park, and G. Moore, "Seaborne measurements of near infrared water-leaving reflectance: The similarity spectrum for turbid waters," Limnology and Oceanography 51, 11671179 (2006).

12. S. G. H. Simis and J. Olsson, "Unattended processing of shipborne hyperspectral reflectance measurements," Remote Sensing of Environment 135, 202-212 (2013).

13. P. M. M. Groetsch, P. Gege, S. G. H. Simis, M. A. Eleveld, and S. W. M. Peters, "Validation of a spectral correction procedure for sun and sky reflections in above-water reflectance measurements," Optics Express 25, 1-12 (2017).

14. M. O. Román, C. B. Schaaf, P. Lewis, F. Gao, G. P. Anderson, J. L. Privette, A. H. Strahler, C. E. Woodcock, and M. Barnsley, "Assessing the coupling between surface albedo derived from MODIS and the fraction of diffuse skylight over spatially-characterized landscapes," Remote Sensing of Environment 114, 738-760 (2010).

15. S. Cui, X. Zhen, Z. Wang, S. Yang, W. Zhu, X. Li, H. Huang, and H. Wei, "Toward a new radiative-transfer-based model for remote sensing of terrestrial surface albedo," Optics Letters 40, 3842-3845 (2015).

16. P. Gege, "Analytic model for the direct and diffuse components of downwelling spectral irradiance in water." Applied optics 51, 14071419 (2012).

17. R. E. Bird and C. Riordan, "Simple Solar Spectral Model for Direct and Diffuse Irradiance on Horizontal and Tilted Planes at the Earth's Surface for Cloudless Atmospheres," Journal of climate and applied meteorology 25, 87-97 (1986).

18. C. A. Gueymard, "Parameterized transmittance model for direct beam and circumsolar spectral irradiance," Solar Energy 71, 325-346 (2001).

19. P. Gege and P. Groetsch, "A spectral model for correcting sun glint and sky glint," in "Proceedings of Ocean Optics XXIII," (2016).

20. D. N. H. Horler, M. Dockray, and J. Barber, "The red edge of plant leaf reflectance," International Journal of Remote Sensing 4, 273-288 (1983).

21. Z. Lee, Y.-h. Ahn, C. Mobley, and R. Arnone, "Removal of surfacereflected light for the measurement of remote-sensing reflectance from an above-surface platform," Optics Express 18, 171-182 (2010).

22. P. M. M. Groetsch, "Sky Reflectance Adjacency Model," http://doi.org/10.5281/zenodo.836756 (2017). 